\author{
Joanna Peradzyńska', Katarzyna Krenke², Anna Szylling², Beata Kołodziejczyk², Agnieszka Gazda ${ }^{3}$, \\ Lidia Rutkowska-Sak', Marek Kulus² \\ 'Department of Epidemiology, Medical University of Warsaw, Poland \\ ${ }^{2}$ Department of Pediatric Pneumonology and Allergy, Medical University of Warsaw, Poland \\ ${ }^{3}$ Department of Pediatric Rheumatology, Institute of Rheumatology, Warsaw, Poland
}

\title{
Abnormalities in lung volumes and airflow in children with newly diagnosed connective tissue disease
}

The authors declare no financial disclosure.

\begin{abstract}
Introduction: Connective tissue diseases (CTDs) of childhood are rare inflammatory disorders, involving various organs and tissues including respiratory system. Pulmonary involvement in patients with CTDs is uncommon but may cause functional impairment. Data on prevalence and type of lung function abnormalities in children with CTDs are scarce. Thus, the aim of this study was to asses pulmonary functional status in children with newly diagnosed CTD and follow the results after two years of the disease course.

Material and methods: There were 98 children (mean age: $13 \pm 3 ; 76$ girls), treated in Department of Pediatric Rheumatology, Institute of Rheumatology, Warsaw and 80 aged-matched, healthy controls (mean age 12.7 $\pm 2.4 ; 50$ girls) included into the study. Study procedures included medical history, physical examination, chest radiograph and PFT (spirometry and whole body-plethysmography). Then, the assessment of PFT was performed after 24 months.

Results: $\mathrm{FEV}_{1}, \mathrm{FEV}_{1} / \mathrm{FVC}$ and MEF50 were significantly lower in CTD as compared to control group, there was no difference in FVC and TLC. The proportion of patients with abnormal lung function was significantly higher in the study group, 41 (42\%) vs 9 (11\%). 24-months observation didn't reveal progression in lung function impairment.

Conclusions: Lung function impairment is relatively common in children with CTDs. Although restrictive ventilatory pattern is considered typical feature of lung involvement in CTDs, airflow limitation could also be an initial abnormality.
\end{abstract}

Key words: lung function tests, rheumatic diseases of childhood, pulmonary involvement

Pneumonol Alergol Pol 2016; 84: 22-28

\section{Introduction}

Connective tissue diseases (CTDs) of childhood are rare inflammatory autoimmune disorders affecting most commonly joints, muscles and skin. In many cases they are related to other organs manifestation, including the eyes, heart, lungs, kidneys, gastrointestinal tract, and blood vessels [1]. Pulmonary complications are infrequent in the childhood CTDs but may cause functional impairment and increase mortality
[2]. Moreover CTDs treatment may also affect respiratory tract. Pulmonary involvement in children may be symptomatic or clinically silent and manifests only as an abnormal diagnostic tests. Although rare, but most commonly observed is diffuse parenchymal lung disease which can be observed in almost every condition from the spectrum of rheumatic diseases. Other include pleurisy, typical for systemic lupus erythematosus (SLE) and juvenile idiopathic arthritis (JIA), pulmonary hemorrhage, shrinking lung syndrome

Address for correspondence: Katarzyna Krenke, Department of Pediatric Pneumonology and Allergy, Medical University of Warsaw, Żwirki i Wigury 63A, 02-091 Warsaw, Poland, e-mail: katarzynakrenke@gmail.com, Tel./Fax: +48 223179419

DOI: 10.5603/PiAP.a2015.0081

Received: 27.08 .2015

Copyright (C) 2015 PTChP

ISSN 0867-7077 
or pulmonary arterial hypertension, which is major cause of mortality in systemic scleroderma and mixed connective tissue disease [3]. In localized scleroderma, considered as a disease of the skin and subcutaneous tissue, respiratory involvement is also reported. The prevalence and clinical features of extracutaneus manifestations in a large cohort of children (750 patients) were confirmed in $22.4 \%$ patients, and respiratory involvement in $2.6 \%$ of them [4].

In adults, involvement of the respiratory system is a common manifestation of CTD. Although some characteristic clinical and radiological patterns are recognized, the respiratory system may be involved in all its components: airways, vessels, parenchyma, pleura, respiratory muscles. Pulmonary symptoms in a patient with a known CTD require a prompt and systematic work-up, thus the diagnosis include wide range of procedures as imaging studies, bronchoscopy, lung function tests and sometimes lung biopsy [5].

Detection of pulmonary complication in children with CTDs is based on the same procedures as in adults, but for some reasons is more complicated. Diagnostic process include basic chest radiography, and more relevant high resolution computed tomography (HRCT). Although very sensitive technique but related to high doses of radiation. The other, invasive procedure is bronchoalveolar lavage, indicated only in cases with respiratory symptoms and signs. The widely used screening method is pulmonary function tests (PFTs), however in pediatric population related to some limitations caused by the lack of a child-investigator cooperation. Since it is non-invasive and relatively cheap, PFTs are invaluable as a screening test of general respiratory health and are extensively used procedure in the diagnostic process in many conditions, which can affect respiratory system, even in asymptomatic patients. Albeit respiratory function in adults with CTD has been extensively investigated, relatively small number of studies including small groups of patients have been carried out in children [6-13]. Thus, the authors conducted this study to asses pulmonary functional status in children with newly diagnosed CTD and follow the results after two years of the disease course.

\section{Material and methods}

\section{Study population}

Ninety-eight children (mean age: $13 \pm 3 ; 76$ girls and 22 boys), treated in Department of Pediatric Rheumatology, Institute of Rheumatology,
Warsaw and 80 aged-matched, healthy control subjects (mean age $12.7 \pm 2.4 ; 50$ girls and 30 boys) were enrolled into the study.

Diagnosis of CTD was made according to commonly accepted criteria. These included: American College of Rheumatology Classification Criteria for systemic lupus erythematosus (year 1982, revised in 1997) and American College of Rheumatology Criteria for systemic scleroderma $(1980)[14,15]$. Nelson criteria were used to diagnose localized scleroderma (LS) and Kasukawa's criteria (1988) to diagnose mixed connective tissue disease (MCTD) [16, 17]. Diagnosis of juvenile dermatomyositis (JDM) was based on Bohan and Peter criteria from 1975 and 2006 international consensus [18, 19]. Scleromyositis (SCL) was diagnosed in patients presenting symptoms of both: SS and polymyositis and in whom serological marker anti-PM/Scl was detected. Diagnosis of undifferentiated connective tissue disease (UCTD) required demonstration of 1) signs and symptoms consistent with CTDs but not fulfilling the classification criteria for any defined CTDs and 2) detection of antinuclear antibodies in serum for at least 3 years.

Study group consisted of: 30 children with LS and 14 with SS, 22 with UCTD, 14 children with SCL, 14 children with MCTD, 2 with DM, and 2 children with SLE.

The control group was recruited from the healthy school children. Children with any respiratory signs and symptoms were excluded.

\section{Study protocol}

This prospective observational study was performed between January 2006-December 2010. All patients entered the study within 4 weeks from the diagnosis of CTD and were followed-up for at least 24 months. Initial evaluation performed at the time of study entry included medical history, physical examination, chest radiograph and lung function tests.

Then, the course of the disease and management were followed up and second assessment of lung function tests was performed after 24 months.

All patients underwent spirometry with flow-volume curve analysis (Spirometer Lungtest 1000, MES, Poland) as well as whole body-plethysmography (Bodyplethysmograph Lungtest 1000, MES, Poland). Following parameters were measured during lung function testing: forced vital capacity (FVC), forced expiratory volume in 1 second $\left(\mathrm{FEV}_{1}\right)$, maximal expiratory flow at $50 \%$ and $25 \%$ of vital capacity (MEF50 and MEF25, respectively). Total lung capacity (TLC) was measured by body plethysmography. All pulmonary 
Table 1. Lung function results in study and control group presented as z-score

\begin{tabular}{lccc}
\hline & Study group & Control subjects & p \\
\hline Age (year) \pm SD & $13.01 \pm 2.99$ & $12.72 \pm 2.38$ & NS \\
Height (cm) \pm SD & $151.3 \pm 14.9$ & $156.2 \pm 13.9$ & 0.002 \\
$\mathrm{FVC}^{*} \pm \mathrm{SD}$ & $-0.52 \pm 1.7$ & $-0.62 \pm 1.1$ & $\mathrm{NS}$ \\
$\mathrm{FEV}_{1}{ }^{*} \pm \mathrm{SD}$ & $-1.56 \pm 1.7$ & $-0.51 \pm 1.1$ & 0.000 \\
$\mathrm{FEV}_{1} / \mathrm{FVC}^{*} \pm \mathrm{SD}$ & $-0.98 \pm 1.8$ & $0.24 \pm 1.2$ & 0.000 \\
$\mathrm{MEF50}^{*} \pm \mathrm{SD}$ & $-1.8 \pm 3.0$ & $-0.77 \pm 1.2$ & 0.0049 \\
$\mathrm{MEF} 25^{*} \pm \mathrm{SD}$ & $-0.31 \pm 1.5$ & $-0.19 \pm 0.9$ & 0.058 \\
TLC $^{*} \pm \mathrm{SD}$ & $0.41 \pm 1.9$ & $0.1 \pm 1.8$ & 0.461 \\
\hline
\end{tabular}

$\mathrm{FEV}_{1}$ — forced expiratory volume in 1 second; FVC — forced vital capacity; $\mathrm{FEV}_{1} / \mathrm{FVC}$ ratio; MEF50 — maximal expiratory flow at $50 \%$ of vital capacity; MEF25 — maximal expiratory flow at $25 \%$ of vital capacity; TLC — total lung capacity; NS — not significant; SD — standard deviation; *lung function measures presented as z-scores

function parameters are presented as the number of standard deviations (SD) from reference value (z-score). The lower limit of normal (LLN) for FVC, $\mathrm{FEV}_{1}, \mathrm{FEV}_{1} / \mathrm{FVC}, \mathrm{MEF} 25, \mathrm{MEF} 50$ and TLC was set at $(-1.96)$ SD below the calculated reference value. Similarly, airway obstruction was diagnosed if z-score for $\mathrm{FEV}_{1} / \mathrm{FVC}$ ratio was below $(-1.96)$ SD. Abnormal small airways flow was defined as MEF50 and MEF25 z-score lower than $(-1.96)$ SD. Restrictive ventilatory defect was diagnosed when TLC z score was below $(-1.96)$ SD.

\section{Statistical analysis}

Statistical analysis was performed with STATISTICA software, version 10.0 (StatSoft, Tulsa, OK, USA). Variables were expressed as mean and standard deviation. Differences between variables in two groups were tested with nonparametric Mann-Whitney $U$ test and Kruskal-Wallis ANOVA for more than 2 groups. The Chi-squared test (Fisher's test, Mc Nemar's test) was used to assess the proportions of patients with abnormal results of different parameters in the control and in the study group. Wilcoxon rank test was applied to compare results at the baseline and after 2 years. All $P$ values were 2-tailed and $p<0.05$ was considered statistically significant.

\section{Ethical considerations}

The study protocol was accepted by Institutional Review Board of Medical University of Warsaw.

\section{Results}

\section{Symptoms}

There were no children complaining of dyspnea or exercise limitation. Seven of 98 children
(7\%) presented with chronic cough, 10 (10\%) had recurrent upper respiratory tract infections. Physical examination revealed wheezing in one patient. No other signs of respiratory diseases were found.

\section{Imaging}

The chest $\mathrm{x}$ - ray was abnormal in 2 cases. In both patients discrete pleural thickenings were detected.

\section{Lung function}

The results of spirometry and whole body -plethysmography in both, study and control group are presented in Table 1.

There were differences in $\mathrm{FEV}_{1}, \mathrm{FEV}_{1} / \mathrm{FVC}$ and MEF50 expressed as z-scores in the study group as compared to healthy subjects $(-1.56 \pm 1.7$ vs $-0.51 \pm 1.1 ;-0.98 \pm 1.8$ vs $0.24 \pm 1.2 ;-1.8$ \pm 3.0 vs $-0.77 \pm 1.2$ respectively). The values measured in the study group were significantly lower than the respective values in controls.

Significant differences were also demonstrated in the percentage of patients with the results below the LLN. The proportion of patients with abnormal lung function was significantly higher in the study group than the healthy controls. Functional lung impairment defined as FVC or $\mathrm{FEV}_{1}$ or $\mathrm{FEV}_{1} / \mathrm{FVC}$ below LLN was found in 41 (42\%) children with CTD and only in 9 (11\%) controls. There was only one patient in study group with decreased $\mathrm{FEV}_{1}$ and FVC but with normal TLC. The prevalence of various lung function abnormalities in both groups, including p-values, ORs and CIs are presented in Table 2.

Spirometry results aggregated according to the diagnosis are presented in Table 3. Subgroup analysis showed differences for FVC ( $p=0.041)$ but not for TLC. Further statistical analysis - 
Table 2. Abnormal lung function results observed in study and control group

\begin{tabular}{|c|c|c|c|c|}
\hline \multirow[b]{2}{*}{ Parameter } & \multicolumn{2}{|c|}{ Number (percentage) of the results below LLN } & \multirow[b]{2}{*}{ Chi-square } & \multirow[b]{2}{*}{$\mathbf{O R}$ (CI) } \\
\hline & Study group & Control group & & \\
\hline FVC & $14(14)$ & $7(9)$ & NS & NA \\
\hline $\mathrm{FEV}_{1}$ & $35(36)$ & $7(9)$ & $p=0.00$ & $\begin{array}{c}\text { OR } 5.79 \\
(2.41-13.95)\end{array}$ \\
\hline $\mathrm{FEV}_{1} / \mathrm{FVC}$ & $27(28)$ & $2(3)$ & $p=0.00$ & $\begin{array}{c}\text { OR } 14.31 \\
(3.4-64.62)\end{array}$ \\
\hline MEF 50 & $34(35)$ & $13(17)$ & $p=0.0055$ & $\begin{array}{c}\text { OR } 2.74 \\
(1.32-5.65)\end{array}$ \\
\hline MEF 25 & $11(12)$ & $0(0)$ & $p=0.02$ & \\
\hline TLC & $5(6)$ & $2(3)$ & NS & NA \\
\hline
\end{tabular}

SD - standard deviation; OR — odds ratio; $\mathrm{Cl}$ - confidence interval; NS - not significant; LLN - lower limit of normal; FEV ${ }_{1}$ - forced expiratory volume in 1 second; FVC — forced vital capacity; FEV $/$ FVC ratio; MEF50 - maximal expiratory flow at $50 \%$ of vital capacity; MEF25 — maximal expiratory flow at $25 \%$ of vital capacity; TLC — total lung capacity

Table 3. Age and lung function tests results in study group according to the diagnosis of CTD

\begin{tabular}{lcccccccc}
\hline & UCTD & MCTD & LS & SS & SCL & DM & SLE & p \\
\hline \multicolumn{1}{c}{$\mathbf{N}$} & $\mathbf{2 2}$ & $\mathbf{1 4}$ & $\mathbf{3 0}$ & $\mathbf{1 4}$ & $\mathbf{1 4}$ & $\mathbf{2}$ & $\mathbf{2}$ & \\
\hline AGE (years) $\pm \mathrm{SD}$ & $14.8 \pm 1.7$ & $13.5 \pm 2.7$ & $11.2 \pm 3.2$ & $13.2 \pm 2.7$ & $12.6 \pm 2.9$ & $17.0 \pm 1.2$ & $13.5 \pm 0.7$ & 0.009 \\
$\mathrm{FVC}^{*} \pm \mathrm{SD}$ & $-0.7 \pm 1.7$ & $-0.46 \pm 2.2$ & $0.12 \pm 1.5$ & $-0.8 \pm 1.5$ & $-0.97 \pm 1.3$ & $1.5 \pm 0.4$ & $-2.4 \pm 1.2$ & 0.041 \\
$\mathrm{FEV}_{1}{ }^{*} \pm \mathrm{SD}$ & $-1.5 \pm 1.5$ & $-1.4 \pm 2.6$ & $-1.3 \pm 1.7$ & $-1.4 \pm 1.6$ & $-1.8 \pm 1.4$ & $-4.3 \pm 0.9$ & $-3.2 \pm 0.7$ & $\mathrm{NS}$ \\
$\mathrm{FEV}_{1} / \mathrm{FVC}^{*} \pm \mathrm{SD}$ & $-0.5 \pm 1.6$ & $-0.9 \pm 1.3$ & $-1.75 \pm 2.0$ & $-0.33 \pm 1.3$ & $-0.5 \pm 1.6$ & $-3.8 \pm 2.2$ & $-0.4 \pm 1.2$ & $\mathrm{NS}$ \\
$\mathrm{MEF50}^{*} \pm \mathrm{SD}$ & $-0.9 \pm 2.0$ & $-2.7 \pm 3.8$ & $-1.4 \pm 2.6$ & $-1.3 \pm 2.1$ & $-1.4 \pm 1.9$ & $-12.0 \pm 2.9$ & $-7.1 \pm 1.1$ & $\mathrm{NS}$ \\
$\mathrm{MEF}^{*} \pm \mathrm{SD}$ & $-0.02 \pm 1.2$ & $-0.6 \pm 1.3$ & $-0.4 \pm 1.6$ & $0.13 \pm 1.3$ & $0.15 \pm 1.1$ & $-0.7 \pm 0.1$ & $-1.7 \pm 0.7$ & $\mathrm{NS}$ \\
$\mathrm{TLC}^{*} \pm \mathrm{SD}$ & $0.39 \pm 1.7$ & $0.4 \pm 2.2$ & $0.8 \pm 2.3$ & $0.3 \pm 1.4$ & $-1.4 \pm 0.9$ & $-0.7 \pm 1.1$ & $-2.0 \pm 1.3$ & $\mathrm{NS}$ \\
\hline
\end{tabular}

UCTD — undifferentiated connective tissue disease; MCTD — mixed connective tissue disease; LS — localized scleroderma; SS — systemic sclerosis; SCL — Scleromyositis; DM — dermatomyositis; SLE — systemic lupus erythematosus; FEV $_{1}$ — forced expiratory volume in 1 second; FVC — forced vital capacity; FEV $/$ FVC ratio; MEF50 - maximal expiratory flow at $50 \%$ of vital capacity; MEF25 - maximal expiratory flow at $25 \%$ of vital capacity; TLC — total lung capacity; NS — not significant; SD — standard deviation; *lung function measures presented as z-scores

multiple group comparisons showed no significant differences.

We did not find any abnormalities in lung function tests in children with chronic cough and recurrent respiratory tract infections.

\section{Patient management and follow-up}

Appropriate pharmacological treatment had been undertaken in 98 children. This included: systemic corticosteroids (28 patients), antimalarials (chloroquine phosphate, hydroxychloroqiune) (29 patients), sulfa drugs (sulfasalazine) (2 patients) nonsteroidal anti-inflammatory drugs (7 patients), chelator (penicylamine) (11 patients), vasodilators (buflomedil hydrochloride, xantinoli nicotinate, pentoxifilline) (27 patients), herbal supplements (piascledine - extract of soybean and avocado) (65 patients) and vitamins (A, E, PP)
(33 patients). The respective therapeutic regimens and drug doses were at the discretion of the attending physicians and were adjusted individually according to disease stage and activity.

After two years of follow-up 89 children underwent second lung function testing. None of the parameters differed significantly when compared to the baseline results.

\section{Discussion}

Our study showed pulmonary functional impairment in children with CTD. The overall proportion of abnormal spirometry results was almost 4-fold higher in our study group than in age-matched healthy controls. This was even more significant in respect to some lung function parameters including $\mathrm{FEV}_{1}$ and $\mathrm{FEV}_{1} / \mathrm{FVC}$. 
Unexpectedly, predominant spirometric abnormalities found in children with CTD were suggestive for decreased airflow rather than for reduced lung volumes. Almost 36\% of patients presented abnormal $\mathrm{FEV}_{1}, 27.5 \%$ had significantly decreased $\mathrm{FEV}_{1} / \mathrm{FVC}$ and $34.7 \%$ showed decreased flow in small airways. This is in contrast to some previous studies which demonstrated mainly restrictive ventilatory pattern $[5,7,20]$. It must be admitted, however, that the majority of data on impaired lung function in CTD comes from studies which were performed in adults.

Although CTDs occur at all ages, the highest prevalence was found in young adults in the age group between 15-40 years, with female-male ratio 6-10: 1 [21]. The prevalence of CTDs in children, especially those below the age of 15 years is significantly lower. Thus, the number of studies on lung disease in children with CTDs is relatively low. As the number of children participating in these studies is usually small and pulmonary involvement in this age group is rare, the data on lung diseases in children with CTDs are relatively scarce. In this context our study which involved relatively large group of patients may add to the existing literature.

In one early study which included 62 children with various CTDs (juvenile rheumatoid arthritis, juvenile dermatomyositis, systemic lupus erythematosus and overlap syndrome), decreased VC and diffusing lung capacity for carbon monoxide (DLCO) were demonstrated in most patients with active disease. While in all studied CTD patients during disease remission PFT results did not significantly differ from normal controls. No case of airway obstruction was detected in study group [7].

Restrictive ventilatory pattern was also found by Trapani et al., who investigated the prevalence of asymptomatic pulmonary involvement in children with juvenile DM. These authors followed a small group of 12 children and showed restrictive pattern of impairment in 5 patients. Two had reduced FVC, FEV 1 , MEF25, DLCO and single-breath alveolar volume $\left(\mathrm{V}_{\mathrm{A}}\right)$; one patient also had reduced MEF50. Two children showed a slight reduction of $\mathrm{V}_{\mathrm{A}}$ [8].

Recently, Alkady et al. reported the results of the study aimed at early diagnosis of cardiac and pulmonary involvement in children with juvenile idiopathic arthritis. Although the study group was relatively large and included 45 children it was still much smaller than our group of 98 patients. It should be emphasized that, similarly to our study, this study included control group. Regar- ding pulmonary function, the authors found that children with juvenile idiopathic arthritis (JIA) were characterized by decreased FVC, peak expiratory flow, maximal inspiratory and expiratory pressure as well as reduced DLCO. Spirometric abnormalities were associated with some features of the disease. These included: rheumatoid factor titer, erythrocyte sedimentation rate, disease duration and the duration of methotrexate use. Significant inverse correlation was reported between the above parameters and pulmonary function measures [9].

A small study conducted by Quezada et al. showed lung involvement in 4 of 14 children with CTDs. Restrictive defect of lung function was demonstrated in one patient with SLE. The authors recommend routine checks of pulmonary function in all patients with rheumatologic diseases even in the absence of respiratory symptoms [10].

Although restrictive ventilatory pattern was a predominant lung function abnormality in above mentioned studies, there are also studies which show that CTDs might be associated with airway obstruction. In multicenter prospective study on juvenile DM, pulmonary function impairment was found in 7 (35\%) of twenty patients. Obstructive and restrictive ventilatory defect was diagnosed in three and four patients, respectively. Three patients had an isolated obstructive ventilatory defect, and two of them did not respond to a short acting beta-2 agonist [22].

Airflow limitations in patients with CTDs were also reported by Lilleby et al. The study was performed in a cohort of 60 young adults (mean age $28.1 \pm 9.5$ years and mean disease duration $12.0 \pm 8.3$ years) with childhood onset SLE. Overall prevalence of abnormal PFT results was 37\% (17/46). FVC was reduced in six patients (10\%), and $\mathrm{FEV}_{1}$ was abnormal in nine (15\%) patients. Obstructive and restrictive abnormality was seen in three (7\%) and one (2\%) patients, respectively [11].

The prevalence of airway involvement in patients with CTDs seems to be closely related to the underlying diseases. It is uncommon in SLE, but in Sjögren syndrome - the prevalence of airway disease is higher than the prevalence of interstitial lung disease. Nevertheless, rare cases of bronchiolitis obliterans with organizing pneumonia (BOOP) have been reported in patients with SLE. BOOP lesions are characterized histologically by inflammatory changes within the distal airways and alveoli associated with nonspecific bronchiolar inflammation [23].

In patients with Sjögren syndrome bronchiolitis and bronchiectasis are the most common 
pulmonary manifestations [24]. In MCTD and systemic sclerosis progressive oesophageal dysmotility increases the risk of aspiration pneumonia, chronic aspiration-associated bronchoconstriction, and/or mild pulmonary fibrosis [25, 26].

Our study showed that in children with CTDs airway obstruction is more common than restrictive ventilatory pattern $(27,5 \%$ vs $6 \%)$. This could rise a question, whether this observation was not confounded by coexistence of asthma. Asthma is the most prevalent chronic disease in children and the most common cause of obstructive ventilatory defect among pediatric population. The concomitance of asthma and CTD has been observed in adult patients. The case-control study from Taiwan showed that asthma and other allergic diseases were two-fold more common in patients with SLE as compared to controls [27]. An Australian study that evaluated comorbidity among senior Australians revealed a common coexistence of arthritis with asthma and bronchitis [28]. Our results in children do not support the association between CTDs and asthma. None of the patients with CTD had a history of asthma. Children with obstructive ventilatory pattern had no dyspnea, exercise limitation, chronic cough or recurrent wheeze. However, we have to admit that reversibility test was not performed. This fact is mentioned as a study limitation. On the other hand, since asthma had not been diagnosed in any of CTD patients during two-year follow up, we might speculate that bronchial obstruction was probably not related to asthma.

In our study group, abnormal lung function was significantly more common than structural changes assessed in chest radiographs. There were no children with radiological signs of interstitial lung diseases and the only abnormalities revealed by chest radiographs were mild pleural thickening demonstrated in two children (2\%). This seems to be consistent with the observations previously made in young adults with CTDs. Lilleby et al. reported low frequency of HRCT abnormalities (8\%). They found micronodules in 4 patients and bronchiectasis in one, but none radiographic evidence of ILD. The authors conclude that asymptomatic children with SLE do not require HRCT investigation [11].

Interestingly, pulmonary function reassessment performed after two years did not revealed difference when compared to baseline results. This was also the case in the study by Cerveri et al. who compared baseline and follow-up results of pulmonary function tests in 15 children with
SLE. The mean time interval between the baseline and repeated tests was 4,5 years. The authors observed even improving trends in lung functions. Therefore, they concluded that the presence of early isolated functional abnormalities was not associated with subsequent development of lung disease [12]. As we did not observed any deterioration in the results of pulmonary function tests after two years of follow-up, we may speculate that neither the disease nor the pharmacological treatment negatively affected lung function. However, we realize that this observation could have been biased by relatively short time of follow up.

We are aware about several limitations of our study. First, significant heterogeneity of our study group should be mentioned. This refers not only to wide spectrum of CTDs but also uneven distribution of different clinical entities. Nearly one third of patients suffered from localized scleroderma, while there were only $2 \%$ of patients with SLE and DM. Thus, although we analyzed the results in the context of underlying diseases we believe this is not justified to draw unequivocal conclusions. Second, our pulmonary function assessment has not included DLCO measurement. Decreased DLCO is regarded as an early marker of interstitial lung involvement. Reduced DLCO has been demonstrated in earlier studies in patients with CTDs [29]. Third, reversibility of airflow limitation has not been tested in patients with obstructive ventilatory pattern. Measurement of airflow parameters after inhalation of bronchodilators could have allowed for more in-depth analysis of the nature of bronchial obstruction. Finally, two years follow-up might have been too short to demonstrate CTD associated deterioration in lung function.

\section{Conclusions}

In conclusion, lung function impairment is relatively common in children with CTDs. Thus, these patients should undergo pulmonary function assessment at the time of CTD diagnosis and further in the course of the disease. Although restrictive pattern of lung function is considered as typical feature of lung involvement in CTDs, airflow limitation could also be an initial abnormality. This observation warrants further studies.

\section{Conflict of interest}

The authors declare no conflict of interest. 


\section{References:}

1. Soeb JB. Rheumatic Diseases. In: Hay Jr. WW, Levin MJ, Deterding RR, Abzug MJ, Sondheimer JM (ed.). Current diagnosis and treatment pediatrics. 21 ed., McGraw-Hill Companies 2012; 889-896.

2. Hashkes PJ, Wright BM, Lauer MS et al. Mortality outcomes in pediatric rheumatology in the US. Arthritis Rheum 2010; 62: 599-608. doi: 10.1002/art.27218.

3. Rabinovich CE. Pulmonary complications of childhood rheumatic disease. Paediatr Respir Rev 2012; 13: 29-36. doi: 10.1016/j.prrv.2011.05.005

4. Zulian F, Vallongo C, Woo $\mathrm{P}$ et al. Localized scleroderma in childhood is not just a skin disease. Arthritis Rheum 2005; 52: $2873-2881$.

5. Crestani B. The respiratory system in connective tissue disorders. Allergy 2005; 60: 715-734.

6. Sanner H, Aaløkken TM, Gran JT, Sjaastad I, Johansen B, Flatø B. Pulmonary outcome in juvenile dermatomyositis: a casecontrol study. Ann Rheum Dis 2011; 70: 86-91. doi: 10.1136/ ard.2010.131433

7. Cerveri I, Bruschi C, Ravelli A et al. Pulmonary function in childhood connective tissue diseases. Eur Respir J 1992; 5: 733-738.

8. Trapani S, Camiciottoli G, Vierucci A, Pistolesi M, Falcini F. Pulmonary involvement in juvenile dermatomyositis: a two -year longitudinal study. Rheumatology 2001; 40: 216-220.

9. Alkady EA, Helmy HA, Mohamed-Hussein AA. Assessment of cardiac and pulmonary function in children with juvenile idiopathic arthritis. Rheumatol Int 2012; 32: 39-46. doi: 10.1007/ s00296-010-1548-5

10. Quezada A, Ramos S, Garcia M, Norambuena X, Pavon D. Lung involvement in rheumatologic diseases in children. Allergol Immunopathol (Madr) 2012; 40: 88-91. doi: 10.1016/j. aller.2011.02.009

11. Lilleby V, Aalokken TM, Johansen B, Forre O. Pulmonary involvement in patients with childhood-onset systemic lupus erythematosus. Clin Exp Rheumatol 2006; 24: 203-208.

12. Cerveri I, Fanfulla F, Ravelli A et al. Pulmonary function in children with systemic lupus erythematosus. Thorax 1996; 51: $424-428$.

13. Panigada S, Ravelli A, Silvestri M et al. HRCT and pulmonary function tests in monitoring of lung involvement in juvenile systemic sclerosis. Pediatr Pulmonol 2009; 44: 1226-1234 doi: 10.1002/ppul.21141.

14. Updating the American College of Rheumatology revised criteria for the classification of systemic lupus erythematosus. Arthritis Rheum 1997; 40: 1725.

15. Preliminary criteria for the classification of systemic sclerosis (scleroderma). Subcommittee for scleroderma criteria of the American Rheumatism Association Diagnostic and Therapeutic Criteria Committee. Arthritis Rheum 1980 May; 23: $581-590$.

16. Nelson A. Localized Scleroderma including morphea, linear scleroderma, and eosinophilic fascitis. Curr Probl Pediatr 1996; 26: 318-324.

17. Kasukawa R, Tojo T, Miyawaki S. Preliminary diagnostic criteria for classification of mixed connective tissue disease. In Kasukawa R, Sharp G (ed.). Mixed connective tissue disease and antinuclear antibodies. Elsevier 1987; 41.

18. Bohan A, Peter JB. Polymyositis and dermatomyositis (first of two parts). N Engl J Med 1975; 292: 344-347.

19. Brown VE, Pilkington CA, Feldman BM, Davidson JE. An international consensus survey of the diagnostic criteria for juvenile dermatomyositis (JDM). Rheumatology (Oxford) 2006; 45: 990-993.

20. Marie I, Hachulla E, Cherin P et al. Interstitial lung disease in polymyositis and dermatomyositis. Arthritis Rheum 2002; 47: $614-622$.

21. Gaubitz M. Epidemiology of connective tissue disorders. Rheumatology (Oxford) 2006; 45 Suppl 3: iii3-4.

22. Pouessel G, Deschildre A, Le Bourgeois M et al. The lung is involved in juvenile dermatomyositis. Pediatr Pulmonol 2013; 48: 1016-1025. doi: 10.1002/ppul.22742.

23. .Min JK, Hong YS, Park SH et al. Bronchiolitis obliterans organizing pneumonia as an initial manifestation in patients with systemic lupus erythematosus. J Rheumatol 1997; 24 2254-2257.

24. Kreider M, Highland K. Pulmonary involvement in Sjogren syndrome. Semin Respir Crit Care Med 2014; 35: 255-264. doi: 10.1055/s-0034-1371529.

25. Mier RJ, Shishov M, Higgins GC et al. Pediatric-onset mixed connective tissue disease. Rheum Dis Clin North Am 2005; 31: $483-496$.

26. Castelino FV, Varga J. Interstitial lung disease in connective tissue diseases: evolving concepts of pathogenesis and management. Arthritis Res Ther 2010; 12: 213. doi: 10.1186/ ar3097.

27. Hsiao Y-P, Tsai J-D, Muo C-H et al. Atopic diseases and systemic lupus erythematosus: an epidemiological study of the risks and correlations. Int J Environ Res Public Health 2014; 11: 8112-8122. doi:10.3390/ijerph110808112.

28. Islam MM, Valderas JM, Yen L et al. Multimorbidity and comorbidity of chronic diseases among the senior Australians: prevalence and patterns. PLoS One. 2014; 9: e83783. doi 10.1371/journal.pone.0083783. eCollection 2014.

29. Trad S, Huong du LT, Frances C et al. Impaired carbon monoxide diffusing capacity as a marker of limited systemic sclerosis. Eur J Intern Med 2011; 22: e80-86. doi: 10.1016/j ejim.2011.05.007 